\title{
Comparaison du potentiel insecticide des huiles essentielles de Ocimum gratissimum L. et de Ocimum canum Sims sur Pectinophora gossypiella Saunders (Lepidoptera : Gelechiidae), insecte ravageur du cotonnier en Côte d'Ivoire
}

Koffi Christophe Kobenan

Université Félix Houphouët-Boigny (UFHB),

Laboratoire de Physiologie Végétale, Côte d'Ivoire

Vama Etienne Tia

Université Péléforo Gbon-Coulibaly (UPGC), Côte d'Ivoire

Germain Elisabeth Cynthia Ochou

Université Nangui Abrogoua (UNA), Côte d'Ivoire

Malanno Kouakou

Kouadio Kra Norbert Bini

Centre National de Recherche Agronomique (CNRA), Côte d'Ivoire

Mamadou Dagnogo

Université Nangui Abrogoua (UNA), Côte d'Ivoire

Acka Emmanuel Dick

Université Félix Houphouët-Boigny (UFHB), Côte d'Ivoire

Ochou Germain Ochou

Centre National de Recherche Agronomique (CNRA), Côte d'Ivoire

Doi: 10.19044/esj.2018.v14n21p286 URL:http://dx.doi.org/10.19044/esj.2018.v14n21p286

\begin{abstract}
The abusive use of synthetic pesticides in the phytosanitary protection of cotton in Côte d'Ivoire threatens the viability of the production system. The search for alternative control methods is therefore necessary. Phytosanitary strategies involving plant-based biopesticides have been explored. The insecticidal activity of essential oils of plant species of the genus Ocimum has been the subject of numerous investigations. The objective of this study is to compare the insecticidal potential of two species of the same genus Ocimum gratissimum and $O$. canum on a major cotton pest, the pink worm Pectinophora gossypiella. Adults of the insect were exposed in the laboratory to the toxic effect of different concentrations of these oils by topical application using a micro applicator. The lethal concentrations causing
\end{abstract}


respectively $50 \%\left(\mathrm{LC}_{50}\right)$ and $90 \%\left(\mathrm{LC}_{90}\right)$ death in the populations tested were determined for each of the essential oils. The oil extracted from $O$. gratissimum was the most toxic with $\mathrm{LC}_{50}$ and $\mathrm{LC}_{90}$ respective values of 1.01 $\%$ and $5.05 \%$ as compared to $O$. canum $\left(\mathrm{LC}_{50}=11.33 \%\right.$ and $\mathrm{LC}_{90}=30.04$ $\%$ ). The high toxicity of $O$. gratissimum may be explained by the presence of $24.57 \%$ thymol and $37.79 \%$ p-cimene in its chemical composition. The extract of $O$. gratissimum has shown in laboratory the highest potentiel insecticidal activity, it's field use can therefore be suggested for the control of this cotton pest.

Keywords: Cotton, essential oils, Pectinophora gossypiella, Ocimum gratissimum, Ocimum canum

\section{Résumé}

L'utilisation abusive des pesticides de synthèse dans la protection phytosanitaire du cotonnier en Côte d'Ivoire menace la viabilité du système de production. La recherche de méthodes de lutte alternatives s'avère donc nécessaire. Des stratégies phytosanitaires impliquant les biopesticides à base de végétaux ont été explorées. L'activité insecticide des huiles essentielles des espèces végétales du genre Ocimum a fait l'objet de nombreuses investigations. La présente étude a pour objectif de comparer le potentiel insecticide de deux espèces du même genre Ocimum gratissimum et $O$. canum sur un important ravageur du cotonnier, le ver rose Pectinophora gossypiella. Les adultes de l'insecte ont été exposés, au laboratoire, à l'effet toxique de différentes concentrations de ces huiles par application topique au moyen d'un micro applicateur. Les concentrations létales causant respectivement la mort de $50 \%\left(\mathrm{CL}_{50}\right)$ et de 90\% (CL90) des populations testées, ont été déterminées pour chacune des huiles essentielles. L'huile extraite de $O$. gratissimum a été la plus toxique avec des valeurs respectives de $\mathrm{CL}_{50}$ et $\mathrm{CL}_{90}$ de $1,01 \%$ et 5,05\% par rapport à $O$. canum $\left(\mathrm{CL}_{50}=11,33 \%\right.$ et $\mathrm{CL}_{90}=30,04$ $\%$ ). La toxicité élevée de $O$. gratissimum peut s'expliquer par la présence de $24,57 \%$ de thymol et de $37,79 \%$ de p-cymène dans sa composition chimique. L'extrait de $O$. gratissimum a montré en laboratoire l'activité insecticide potentielle la plus élevée, son utilisation en plein champ peut donc être suggérée pour la lutte contre ce ravageur du cotonnier.

Mots clés : Cotonnier, huiles essentielles, Pectinophora gossypiella, Ocimum gratissimum, Ocimum canum

\section{Introduction}

En Côte d'Ivoire, l'utilisation des produits chimiques pour le contrôle des ravageurs reste la methode de lutte la plus utilisées en culture cotonnière. 
Cependant, l'usage abusif des pesticides de synthèses pour la protection phytosanitaire du cotonnier comporte de nombreux risques pour la santé humaine et pour l'environnement (Konan et al., 2007; Traoré et al., 2008).

En plus, ces dernières années, plusieurs études ont revelé d'importants changements dans le faciès parasitaire du cotonnier. Ces changements se traduisent par la résistance de certains Lépidoptères (Helicoverpa armigera, Thaumatotibia leucotreta, Pectinophora gossypiella) vis à vis des insecticides à base de Pyréthrinoïdes (Martin et al., 2000; Doffou, 2012) et l'emergence des insectes piqueurs-suceurs (Koné et al., 2017; Didi et al., 2018). La recherche de methodes de lutte alternatives à la lutte chimique s'avère donc necessaire.

Ainsi, plusieurs travaux, démontrant le potentiel insecticide des plantes, ont été réalisés (Deguine et al., 2000; Akantetou et al., 2011; Fening et al., 2014; Diabaté et al., 2014; Traoré et al., 2015). Recemment, Yarou et al. (2017) ont fait ressortir de leur revue bibliographique, que les plantes du genre Ocimum (Ocimum canum, Ocimum gratissimum, Ocimum sanctum) étaient les mieux indiquées pour leur capacité biocide et pour leur usage thérapeutique, médicinal et alimentaire. En côte d'Ivoire, diverse études réalisées ont mis en exergue, l'efficacité biologique des extraits de plantes du genre Ocimum (Johnson et al. (2006), Soro et al. (2011), Doumbouya et al. (2012) et Kassi et al. (2014)).

L'objectif principal de la présente étude est de comparer, in vitro, l'activité insecticide de l'huile essentielle de $O$. gratissimum et de $O$. canum sur Pectinophora gossypiella, un important ravageur carpophage du cotonnier. Il s'agira aussi de caractériser les constituants chimiques qui permettraient d'expliquer le potentiel insecticide des huiles essentielles de ces deux plantes aromatiques locales.

\section{Matériel et méthodes}

\section{Site d'expérimentation}

L'étude a été réalisée au Laboratoire d'entomologie du programme de recherche sur le coton du Centre National de Recherche Agronomique (CNRA) sis à Bouaké ( $7^{\circ} 40^{\prime}$ de latitude Nord et $5^{\circ} 2^{\prime}$ de longitude Ouest).

\section{Matériel animal}

Les papillons de $P$. gossypiella, fraîchement sortis de leurs cocons, âgés d'un jour après émergence, ont été utilisés pour les tests biologiques (Doffou, 2012). En effet, les larves de cet insecte étant de très petite taille il serait donc difficile de les traiter par application topique. Les papillons utilisés sont issus des larves collectées des parcelles de cotonniers non traitées implantées dans la localité de Bouaké et qui ont été mises en élevage au Laboratoire. 


\section{Huiles essentielles}

L'étude a été réalisée avec les huiles essentielles extraites des feuilles de deux (02) plantes aromatiques locales appartenant à la famille des Lamiacées. Il s'agit de Ocimum gratissimum L. et de Ocimum canum Sims collectées respectivement dans les régions de Bouaké et de Korhogo, en Côte d'Ivoire. Ces huiles essentielles ont été fournies par le Laboratoire de Biochimie de l'Université Péléféro Gon Coulibaly de Korhogo (Côte d'Ivoire).

\section{Méthodologie}

\section{Extraction et analyses des huiles essentielles}

Les feuilles des deux espèces ont été séchées à la température ambiante du Laboratoire $\left(28 \pm 2^{\circ} \mathrm{C}\right)$ pendant une semaine avant l'extraction d'huile. Les huiles esssentielles ont été obtenues par distillation des feuilles séchées par la méthode de l'entraînement à la vapeur d'eau réalisé avec un alambic en inox (250 L) pendant $3 \mathrm{~h}$. Elle consiste en une distillation classique dans un alambic porté à ébullition dans lequel la matière végétale n'est pas en contact avec l'eau. La vapeur d'eau, au passage dans la matière végétale se charge de composés volatiles et se condense à l'intérieur d'un réfrigérant. Les huiles essentielles, moins denses que l'eau, sont recueillies par simple décantation à la surface de celle-ci (Tia et al., 2013). Les huiles essentielles recueillies sont laissées au repos dans une ampoule à décanter pendant une (1) heure à l'abri de la lumière en vue d'éliminer toute trace d'eau. Elles sont ensuite stockées dans des flacons en verre coloré et conservées à $4{ }^{\circ} \mathrm{C}$ dans un réfrigérateur à l'abri de la lumière jusqu'à leur utilisation.

Les huiles essentielles obtenues à partir des feuilles séchées de $O$. gratissimum et de $O$. canum ont été analysées par Chromatographie en phase gazeuse (CPG) couplée d'une Spectrométrie de Masse sélective (CPG/SM) au Département de Génie Chimique et Agroalimentaire (GCAA) de l'Institut National Polytechnique-Houphouet Boigny (INP-HB) de Yamoussoukro. L'analyse des constituants chimiques a été réalisée à l'aide d'un chromatographe de type Agilent technologies 6890 N Network GC System version N.04.07 équipé d'une colonne capillaire en silice fondue HP- 1 (phényl-méthyl polysiloxane) $(25 \mathrm{~m}$ x $200 \mu \mathrm{m} \times 0,33 \mu \mathrm{m})$. Le four était programmé à une température variant entre 50 à $150^{\circ} \mathrm{C}$ à un gradient de $5^{\circ}$ $\mathrm{C} / \mathrm{min}$. Le gaz vecteur était 1'hélium avec un débit de $0,8 \mathrm{ml} / \mathrm{min}$. Les températures de l'injecteur (Agilent Technologies 7683) et le détecteur (Agilent 5973 Network) étaient respectivement $250^{\circ} \mathrm{C}$ et $280^{\circ} \mathrm{C}$. Un volume de $1 \mu$ d'échantillon d'huile essentielle dilué dans du diéthylether a été injecté en mode split.

Les différents constituants volatils ont été identifiés par leurs spectres de masse et leurs indices de rétention en comparaison avec ceux des composés 
existant de la banque de données. La confirmation des composés a été faite par comparaison (Tia et al., 2013) aux données standard de référence existant dans la banque de données de Nist 98.1 et de wiley275. Les pourcentages relatifs des différents constituants ont été obtenus par intégration de leurs pics sur le spectre du chromatographe en phase gazeuse.

\section{Obtention des papillons}

Les papillons ont été obtenus à partir des larves maintenues en élevage au Laboratoire dans des boîtes en plastique $(50 \mathrm{~cm}$ x $20 \mathrm{~cm})$ contenant de la fibre de coton. Elles ont été soumises à une photopériode de douze heures, une température de $26 \pm 2{ }^{\circ} \mathrm{C}$ et une humidité relative de $70 \pm 5 \%$ (Patana, 1977). Après une semaine, les larves incubées se chrysalident et la nymphose dure en moyenne 13 jours avant l'émergence des papillons.

\section{Tests Biologiques}

\section{Préparation des concentrations d'huile essentielle}

La gamme de concentrations a été déterminée sur la base de plusieurs tests préliminaires au Laboratoire. Ainsi, pour chacune des deux huiles essentielles, l'extrait dilué au 1/80 ème avec de l'acétone (solution à $80 \%$ ) a servi à la préparation des différentes concentrations testées: 0,$25 ; 0,50 ; 1 ; 2$; $4 ; 8 ; 16 ; 32$; et $64 \%$.

\section{Réalisation des bioessais}

Pour les tests, les papillons de même âge ( 1 jour après émergence) ont été répartis par lot de 10 individus et trois répétitions ont été réalisées pour chaque concentration, soit 3 boîtes x 10 individus. Après la constitution des lots, tous les papillons d'un même lot ont été endormis à l'aide du dioxyde de carbone à un débit fixé à $20 \mathrm{l} / \mathrm{min}$ pendant 10 secondes afin de faciliter leur traitement par application topique. Chaque individu a reçu un (1) $\mu$ le solution sur sa partie dorsale opposée à son thorax au moyen d'un micro applicateur Arnold Micro (Burkard, UK) (Kaan et al., 2016). Pour chaque extrait, l'application a été faite dans l'ordre croissant des concentrations préparées. La concentration zéro, constituée uniquement du solvant de dilution (acetone), a servi de témoin absolu (contrôle). Chaque lot d'insectes traités a été immédiatement transféré dans un pondoir $(12 \mathrm{~cm} \times 16 \mathrm{~cm})$ en sachet cellophane. Ils ont été alimentés avec une solution de miel à $10 \%$ (v : v) comme préconisé par Wu et al. (2006) au cours de leur entreposage à la température de $26{ }^{\circ} \mathrm{C} \pm 2{ }^{\circ} \mathrm{C}$ et à $70 \%$ d'humidité relative pour les différentes observations. En somme, les expériences ont porté sur un total de 600 individus (papillons). 


\section{Détermination des taux de mortalité}

Après l'application topique, la mortalité dans les différents lots a été observée à $24 \mathrm{~h}, 48 \mathrm{~h}$ et $72 \mathrm{~h}$. Les insectes immobiles et visiblement moribonds, qui ne peuvent pas voler normalement ont été considérés comme morts. lorsque des insectes morts ou moribonds ont été observés parmi les témoins, les taux de mortalité ont été corrigés par la formule d'Abbott (1925).

$$
\text { Mc }(\%)=\frac{\mathbf{M}_{\mathbf{t}}-\mathbf{M}_{\mathbf{0}}}{100-\mathbf{M}_{\mathbf{0}}} \times 100
$$

Mc : taux de mortalité corrigée

$\mathbf{M}_{0}$ : taux de mortalité dans le lot témoin

Mt : taux de mortalité dans le lot traité

\section{Analyses des données}

Une analyse de variance ou ANOVA a été faite à l'aide du logiciel SPSS 22.0 sur le taux de mortalité corrigé des insectes en fonction des traitements (huiles essentielles et concentrations). En cas de différence significative entre les traitements, la comparaison des moyennes a été effectuée par le test de Duncan au seuil de $5 \%$.

A l'aide du logiciel WinDL 32.0 (CIRAD, Montpellier, version 1998), les concentrations létales causant respectivement la mort de $50 \%$ (CL50) et de 90\% (CL 90$)$ des populations testées ont été déterminées pour chacune des huiles essentielles, à deux jours (48 h) après exposition des papillons.

\section{Résultats}

Compositions chimiques des huiles essentielles de Ocimum gratissimum et de $O$. canum

Les résultats des analyses chimiques des échantillons d'huiles essentielles de $O$. gratissimum et de $O$. canum sont consignés dans le tableau I. Selon les résultats obtenus, l'huile essentielle de $O$. gratissimum est constituée de dix-huit composés représentant $100 \%$ des composants identifiés. Dans le groupe des monoterpènes hydrocarbonés, le $p$ - Cymène avec une teneur de $37,79 \%$ et le Sabinène $(6,60 \%)$ ont constitué les élements terpéniques les plus représentés. Le Thymol (24,57 \%) et le Camphre $(5,53 \%)$ ont occupé une place prépondérante parmi les monoterpènes oxygénés qui sont présents dans cette essence. L'huile essentielle de $O$. gratissimum est aussi composée de sesquiterpènes tels que le $\beta$-cis-Caryophyllène $(4,42 \%)$, le Sélinène $(1,55 \%)$, le Copaène $(1,14 \%)$ et le $\alpha$-Caryophyllène $(0,63 \%)$.

Quant à l'huile essentielle extraite de $O$. canum, l'analyse chimique de celle-ci a mis en exergue une prédominance des composés monoterpéniques oxygénés tels que l'Eucalyptol ou le 1,8 -Cinéol (41,75\%), le Camphre $(16,94 \%)$ et le Myrténal (13,50\%). Cependant, elle est relativement peu 
composée de monoterpènes et en sesquiterpènes hydrocarbonés. L' $\alpha$-Pinène $(5,29 \%)$, le $1 \mathrm{~S}$ - $\alpha$-Pinène $(3,59 \%), \beta$-Pinène $(3,29 \%)$, le $p$ - Cymène $(1,49$ $\%)$, le Camphène $(2,11 \%)$ et $1^{\prime} \alpha$-Caryophyllène $(5,92 \%)$ ont été les seules composantes hydrocarbonées identifiées dans cette huile essentielle. Au total, ce sont douze composés représentant 99,98 \% répartis en trois grands groupes terpéniques, qui ont été identifiés dans cette huile.

Tableau I : Compositions chimiques des huiles essentielles de Ocimum gratissimum et $O$.

canum

\begin{tabular}{|c|c|c|c|}
\hline \multirow{2}{*}{$\begin{array}{c}\text { Groupes } \\
\text { terpéniques }\end{array}$} & \multirow{2}{*}{$\begin{array}{l}\text { Composés } \\
\text { Chimiques }\end{array}$} & \multicolumn{2}{|c|}{ Teneur en pourcentage $(\%)$} \\
\hline & & $\begin{array}{c}\text { Ocimum } \\
\text { gratissimum }\end{array}$ & $\begin{array}{c}\text { Ocimum } \\
\text { canum }\end{array}$ \\
\hline \multirow{12}{*}{$\begin{array}{l}\text { Monoterpènes } \\
\text { hydrocarboné }\end{array}$} & Sabinène & 6,60 & - \\
\hline & $\alpha$-Pinène & 1,86 & 5,29 \\
\hline & 1S- $\alpha$-Pinene & - & 3,59 \\
\hline & $\beta$-Phellandrène & 0,89 & - \\
\hline & $\beta$-Pinène & 0,49 & 3,29 \\
\hline & $\beta$-Myrcène & 3,97 & - \\
\hline & $\alpha$-Terpinène & 1,66 & - \\
\hline & $p$ - Cymène & 37,79 & 1,49 \\
\hline & d-Limonène & 1,26 & - \\
\hline & $\gamma$-Terpinène & 3,62 & - \\
\hline & 2-Nitro-p-Cymene & 2,05 & - \\
\hline & Camphène & - & 2,11 \\
\hline \multirow{7}{*}{$\begin{array}{l}\text { Monoterpènes } \\
\text { oxygénés }\end{array}$} & Terpinène-4-ol & 1,38 & 1,99 \\
\hline & Thymol méthyl éther & 0,59 & - \\
\hline & Thymol & 24,57 & - \\
\hline & Camphre & 5,53 & 16,94 \\
\hline & Bornéol & - & 2,63 \\
\hline & 1,8 Cinéol & - & 41,75 \\
\hline & Myrténal & - & 13,50 \\
\hline \multirow{4}{*}{$\begin{array}{l}\text { Sesquiterpènes } \\
\text { hydrocarbonés }\end{array}$} & Copaène & 1,14 & - \\
\hline & $\beta$-cis-Caryophyllène & 4,42 & - \\
\hline & $\alpha$ - Caryophyllène & 0,63 & 5,92 \\
\hline & Sélinène & 1,55 & - \\
\hline \multirow[t]{2}{*}{$\begin{array}{c}\text { Composé } \\
\text { aromatique }\end{array}$} & Polyphénol éther & - & 1,48 \\
\hline & Total & 100 & 99,98 \\
\hline
\end{tabular}

Effets des huiles essentielles sur la survie de Pectinophora gossypiella Huile essentielle de $\boldsymbol{O}$. gratissimum

Les résultats de l'analyse de variance, présentés dans le tableau 2, indiquent que la mortalité de $P$. gossypiella a été significativement dépendante de la concentration $(\mathrm{p}=0,00)$. En effet, l'évolution de la mortalité des insectes testés s'est faite selon l'augmentation de la concentration de l'huile. Ainsi, 24 $\mathrm{h}$ après l'application, les concentrations de $0,25 \% ; 0,5 \%$; et $2 \%$ ont induit 
des moratilités inférieures à $50 \%$ soient $23,33,26,66$ et 36,66 \% respectivement. Par contre, les concentrations allant de 2 à $64 \%$ ont causé des mortalités comprises entre 63,33 et $100 \%$ à partir du premier jour après le test (24 h) jusqu'à la fin des observation (72 h). Par ailleurs, la concentration minimale de l'huile essentielle de $O$. gratissimum permettant d'obtenir $100 \%$ de mortalité du ravageur a été de $8 \%$. Les valeurs moyennes des taux de mortalité, toutes concentrations confondues, ont été relativement élevées pendant les $72 \mathrm{~h}$ après le test.

\section{Huile essentielle de $\boldsymbol{O}$ canum}

Les résultats des tests de l'activité insecticide de l'huile essentielle de $O$._canum indiquent une relation significative entre les concentrations et les taux de mortalité des adultes de $P$. gossypiella (Tableau III). Pendant les trois périodes d'observation, l'analyse de variance des taux de mortalité en fonction des concentrations, a montré un effet très hautement significatif de cette huile essentielle sur la survie du ravageur $(\mathrm{p}=0,00)$. Toutefois, des neuf concentrations évaluées sur P. gossypiella, il n'y a que trois (16, 32 et $64 \%$ ) qui ont permis d'obtenir un taux de mortalité supérieur à $50 \%$, à chaque observation (24, 48 et 72 h). Les mortalités chez l'insecte ont varié de 72,59 à $76,66 \%$ et de 90,00 à 93,33\%, respectivement avec les concentrations de 16 et $32 \%$ de l'huile essentielle de 0 . canum 72 heures après le test. Par ailleurs, la mortalité de la totalité des insectes testés a été obtenue avec la concentration maximale de $64 \%$. Quant aux concentrations de 1 à $8 \%$, elles n'ont pu tuer $50 \%$ de la population d'insectes testés durant le test.

\section{Comparaison de la toxicité des huiles essentielles de $\boldsymbol{O}$. gratissimum et de O. canum sur $P$. gossypiella}

Les concentrations létales causant respectivement la mort de $50 \%$ (CL50) et de 90\% (CL90) des populations testées, ont été déterminées pour chacune des huiles essentielles. D'après les résultats consignés dans le tableau IV. L'huile extraite de $O$. gratissimum a été largement plus toxique avec des valeurs respectives de: $\mathrm{CL}_{50}=1,01 \%$ et $\mathrm{CL}_{90}=5,05 \%$ en comparaison à celle de O. canum $\left(\mathrm{CL}_{50}=11,33 \%\right.$ et $\left.\mathrm{CL}_{90}=30,04 \%\right)$. En effet, les CL90 et CL50 obtenues avec l'huile essentielle de $O$. gratissimum ont été respectivement six et onze fois moins élevées que celles observées avec l'extrait de $O$. canum.

Tableau II: Evolution chronologique de la mortalité moyenne des adultes de Pectinophora gossypiella en fonction de la concentration d'huile essentielle de Ocimum gratissimum appliquée

\begin{tabular}{cccc}
\hline \multirow{2}{*}{$\begin{array}{c}\text { Concentrations de } \\
\text { l'huile essentielle }\end{array}$} & \multicolumn{3}{c}{ Mortalité moyenne (\%) } \\
\cline { 2 - 4 }$(\boldsymbol{\%})$ & $\mathbf{2 4} \mathbf{h}$ & $\mathbf{4 8 h}$ & $\mathbf{7 2} \mathbf{~ h}$ \\
\hline $\mathbf{6 4}$ & $100,00 \pm 0,00 \mathbf{a}$ & $100,00 \pm 0,00 \mathbf{a}$ & $100,00 \pm 0,00 \mathbf{a}$ \\
$\mathbf{3 2}$ & $100,00 \pm 0,00 \mathbf{a}$ & $100,00 \pm 0,00 \mathbf{a}$ & $100,00 \pm 0,00 \mathbf{a}$
\end{tabular}




\begin{tabular}{cccc}
$\mathbf{1 6}$ & $100,00 \pm 0,00 \mathbf{a}$ & $100,00 \pm 0,00 \mathbf{a}$ & $100,00 \pm 0,00 \mathbf{a}$ \\
$\mathbf{8}$ & $100,00 \pm 0,00 \mathbf{a}$ & $100,00 \pm 0,00 \mathbf{a}$ & $100,00 \pm 0,00 \mathbf{a}$ \\
$\mathbf{4}$ & $86,66 \pm 8,81 \mathbf{a}$ & $90,00 \pm 10,00 \mathbf{a}$ & $90,00 \pm 10,00 \mathbf{a}$ \\
$\mathbf{2}$ & $63,33 \pm 3,33 \mathbf{b}$ & $63,33 \pm 3,33 \mathbf{b}$ & $66,66 \pm 3,33 \mathbf{b}$ \\
$\mathbf{1}$ & $36,66 \pm 8,81 \mathbf{c}$ & $36,66 \pm 8,81 \mathbf{c}$ & $36,66 \pm 14,52 \mathbf{c}$ \\
$\mathbf{0 , 5}$ & $26,66 \pm 6,66 \mathbf{c}$ & $26,66 \pm 6,66 \mathbf{c}$ & $33,33 \pm 3,33 \mathbf{c}$ \\
$\mathbf{0 , 2 5}$ & $23,33 \pm 13,33 \mathbf{c}$ & $23,33 \pm 13,33 \mathbf{c}$ & $26,66 \pm 16,66 \mathbf{c}$ \\
Témoin & $0,00 \pm 0,00 \mathbf{d}$ & $0,00 \pm 0,00 \mathbf{d}$ & $0,00 \pm 0,00 \mathbf{d}$ \\
\hline Moyenne & $63,66 \pm 7,03$ & $64,00 \pm 7,08$ & $65,33 \pm 7,00$ \\
Signification $(p)$ & 0,00 & 0,00 & 0,00 \\
\hline Al'ing
\end{tabular}

A l'intérieur d'une même colonne, les moyennes affectées d'une même lettre ne diffèrent pas statistiquement au seuil de $5 \%$ (test de Duncan).

Tableau III : Evolution chronologique de la mortalité moyenne des adultes de Pectinophora gossypiella selon la concentration d'huile essentielle de Ocimum canum appliquée

\begin{tabular}{|c|c|c|c|}
\hline \multirow{2}{*}{$\begin{array}{l}\text { Concentration de } \\
\text { l'huile essentielle } \\
(\%)\end{array}$} & \multicolumn{3}{|c|}{ Mortalité moyenne (\%) } \\
\hline & $24 \mathrm{~h}$ & $48 h$ & $72 \mathrm{~h}$ \\
\hline 64 & $100 \pm 0,00 \mathbf{a}$ & $100 \pm 0,00 \mathbf{a}$ & $100 \pm 0,00 \mathbf{a}$ \\
\hline 32 & $90,00 \pm 0,00 \mathbf{b}$ & $90,00 \pm 0,00 \mathbf{b}$ & $93,33 \pm 5,77 \mathbf{a}$ \\
\hline 16 & $72,59 \pm 10,96 \mathbf{c}$ & $76,66 \pm 5,77 \mathbf{c}$ & $76,66 \pm 5,77 \mathbf{b}$ \\
\hline 8 & $20,00 \pm 0,00 \mathbf{d}$ & $23,33 \pm 5,77 \mathbf{d}$ & $23,33 \pm 5,77 \mathbf{c}$ \\
\hline 4 & $10,00 \pm 10,00 \mathbf{e}$ & $13,33 \pm 5,77 \mathbf{e}$ & $16,66 \pm 5,77 \mathbf{c d}$ \\
\hline 2 & $3,33 \pm 5,77 \mathbf{e}$ & $6,66 \pm 11,54$ ef & $6,66 \pm 11,54$ de \\
\hline 1 & $3,33 \pm 5,77 \mathbf{e}$ & $3,33 \pm 5,77$ ef & $6,66 \pm 11,54$ de \\
\hline $\mathbf{0 , 5 0}$ & $0,00 \pm 0,00 \mathbf{e}$ & $0,00 \pm 0,00 \mathbf{f}$ & $0,00 \pm 0,00 \mathbf{e}$ \\
\hline 0,25 & $0,00 \pm 0,00 \mathbf{e}$ & $0,00 \pm 0,00 \mathbf{f}$ & $0,00 \pm 0,00 \mathbf{e}$ \\
\hline Témoin & $0,00 \pm 0,00 \mathbf{e}$ & $0,00 \pm 0,00 \mathbf{f}$ & $0,00 \pm 0,00 \mathbf{e}$ \\
\hline Moyenne & $29,92 \pm 7,22$ & $31,33 \pm 7,21$ & $32,33 \pm 7,26$ \\
\hline Signification $(p)$ & 0,00 & 0,00 & 0,00 \\
\hline
\end{tabular}

A l'intérieur d'une même colonne, les moyennes affectées d'une même lettre ne diffèrent pas statistiquement au seuil de $5 \%$ (test de Duncan).

Tableau IV : Concentrations létales ( $\mathrm{CL}_{50}$ ET $\mathrm{CL}_{90}$ en \%) des huiles essentielles de Ocimum gratissimum et $O$. canum $48 \mathrm{~h}$ après application sur les adultes de Pectinophora gossypiella

\begin{tabular}{cccccc}
\hline $\begin{array}{c}\text { Plantes locales } \\
\text { utilisées }\end{array}$ & $\mathbf{N}$ & $\lambda^{\mathbf{2}}$ & ddl & CL50 (\%) & CL90(\%) \\
\hline $\begin{array}{c}\boldsymbol{O} . \\
\text { gratissimum }\end{array}$ & 210 & 8,50 & 4 & $1,01[0,13-1,93]$ & $5,05[3,05-9,75]$ \\
\hline $\begin{array}{c}\boldsymbol{O} . \\
\text { canum }\end{array}$ & 210 & 4,36 & 4 & $11,34[8,69-14,26]$ & $30,39[22,61-51,08]$ \\
\hline
\end{tabular}

$\mathbf{N}$ : nombre d'adultes testés, $\lambda^{2}:$ Chi 2 , ddl : degré de liberté, CL50 : concentration létale à 50 $\%$, CL90 : concentration létale à $90 \%$ 


\section{Discussion}

Les résultats de l'analyse de la composition chimique des huiles essentielles ont revélé que celle extraite de $O$. gratissimum est plus riche en monoterpènes hydrocarbonés contrairement à l'huile de $O$. canum. Ces résultats corroborent ceux obtenus des travaux de Tchoumbougnang et al. (2009), de Akantetou et al. (2011) et de Ouedraogo et al. (2016). En effet, dans les chémotypes étudiés respectivement par ces auteurs, une forte composition en éléments hydrocarbonés a été détectée dans les essences de $O$. gratissimum. Le Para-cymène et le thymol, avec respectivement des proportions de 37,79 et $24,59 \%$, ont été les molécules majoritaires de l'huile essentielle de $O$. gratissimum. Koffi et al. (2013), ont fait ces mêmes constats avec leur chémotype provénant de la localité de Nianda au Sud de la Côte d'Ivoire. La présence remarquable du Thymol dans le présent chémotype étudié est généralement singulier aux extraits de $O$. gratissimum comme le montrent les travaux de recheche de plusieurs auteurs (Ngamo et al., 2001; Gueye et al., 2011; Nguemtchouin, 2012). D'après les analyses chromatographiques, l'huile essentielle du chémotype $O$. canum utilisé a été composée majoritairement de 1,8-Cinéol et de Camphre (41,75 et 13,50\% respectivement). Hassane et al. (2011) ont aussi signalé la présence du 1,8cinéole et du camphre comme composés majoritaires dans les huiles essentielles de leur chémotype qui provenaient de deux localités distinctes du Maroc. Au Bénin, par contre, Akantetou et al. (2011) ont revelé de faible teneur en ces éléments terpéniques.

Les bioessais réalisés ont montré que les huiles essentielles des deux plantes aromatiques ont un effet insecticide sur les adultes de P. gossypiella. Après application, elles ont entrainé, la mort de la majorité des individus testés selon les concentrations utilisées. Ces observations confirment leurs propriétés insecticides en plus de leurs activités antifongiques et antimicrobiennes mises en évidence par des travaux antérieurs (Camara et al., 2010 ; Gonzalez et al., 2013). La forte présence des composées monoterpéniques dans les essences des deux plantes aromatiques locales expliquerait leur efficacité insectide contre les adultes de $P$. gossypiella. En effet, Konstantopoulou et al. (1992) ont révélé que les monoterpènes des huiles essentielles sont toxiques pour de nombreux insectes. Ben Abdelkader (2012) a, par ailleurs, montré que l'efficacité insecticide d'une huile essentielle serait due à la nature et à la structure chimique de ses constituants terpéniques. Les valeurs de $\mathrm{CL}_{50}$ ont montré cependant que l'huile essentielle de $O$. gratissimum est largement plus toxique que celle de $O$. canum sur $P$. gossypiella. Cette potentialité insecticide remarquable de $O$. gratissimum serait due à la présence du thymol comme l'ont prouvée Tchoumbougnang et al. (2009) sur les larves de Anopheles gambiae au Caméroon; puis Koul et al. (2013) sur les larves de Helicoverpa armigera et de Spodoptera litura en Inde; Ouedraogo et al. (2016) sur les 
principaux insectes nuisibles du maïs en stockage au Burkina Faso. D’autres auteurs tels que Cloyd et Chiasson (2007) ont émis l'hypothèse selon laquelle, ce composé oxygéné agirait directement sur la cuticule des insectes et acariens, notamment ceux à corps mou en entrainant sa dégradation. Le thymol interférerait également sur l'activité des synapses, ce qui empêcherait par étouffement la respiration et conduirait à la mort de l'insecte (Priestley et al., 2003 ; Gonzalez et al., 2013).

L'huile essentielle de $O$. canum a eu une toxicité onze fois plus faible sur $P$. gossypiella, comparativement à celle de $O$. gratissimum. Cette faible efficacité pourrait être attribuée à l'abscence du thymol de la composition chimique de cette huile essentielle.

\section{Conclusion}

Les huiles essentielles extraites des deux plantes aromatiques locales (Ocimum canum et $O$ gratissimum) ont des propriétés insecticides sur $P$. gossypiella. En se référant aux valeurs des concentrations létales de chacune de ces huiles utilisées, l'étude a révélé que l'huile essentielle de $O$. gratissimum est la plus toxique. Des essais devraient être envisagés en milieu semi-contrôlé (sous serre) puis en plein champ pour évaluer son efficacité biologique sur les principaux insectes ravageurs du cotonnier. Cela pourrait déboucher sur l'utilisation pratique de ces huiles essentielles comme une solution alternative dans les systèmes intégrés de lutte contre les nuisibles de la culture cotonnière en Côte d'Ivoire. De même, leur application et leur large diffusion auprès des populations paysannes seraient peu onéreuses et respectueuses de l'environnement et la santé des populations.

\section{References:}

1. Abbott, W.S. (1925). Amethod for computing the effectiveness of an insecticide.J. Econ. Entomol. Vol. 18, pp. 265-267.

2. Akantetou, P. K., Koba, K., Nenonene, A. Y., Poutouli, W. P., Raynaud, C. \& Sanda, K. (2011). Evaluation du potentiel insecticide de l'huile essentielle d'Ocimum canum Sims sur Aphis gossypii Glover (Homoptera : Aphididae) au Togo, IJBCS, 5 (4), 1491-1500. https://doi.org/10.4314/ijbcs.v5i4.15.

3. Ben Abdelkader, T. (2012). Biodiversité, bioactivité et biosynthèse des composées terpéniques volatiles des lavandes ailées, Lavandula stoechas sensu lato, un complexe d'espèces méditerranéennes d'intérêt pharmacologique. Thèse de Doctorat: Université Jean Monnet - Saint-Etienne, Ecole Normale Supérieure de Kouba (Alger), p.283. URL : https://tel.archives-ouvertes.fr/tel-00952695/document.

4. Camara, B., Dick, E., Sako, A., Kone, D., Kanko, C., Boye, M.-A.D., Ake, S. \& Anno, A. (2010). Lutte biologique contre Deightoniella 
torulosa (Syd.) Ellis, par l'application des huiles essentielles d'Eucalyptus platyphylla $\mathrm{F}$. Muell. et de Melaleuca quinquenervia $\mathrm{L}$. Phytothérapie et écologie, 8, 240-244. URL: https://doi.org/10.1007/s10298-010-0568-3.

5. Cloyd, R. \& Chiasson, H. (2007). Activity of an essential oil derived from Chenopodium ambrosioides on Greenhouse Insect Pests. J. Econ. Entomol., 100,459-466. URL : http://www.bioone.org/doi/ full $/ 10.1603 / 00220493 \% 282007 \% 29100 \% 5 B 459 \% 3$ AAOAEOD $\% 5$ D2.0.CO \%3B2.

6. Deguine, J-P., Vaissayre, M. (2000). Proposition pour une gestion durable des populations de puceron, d'aleurodes chez les petits producteurs de coton africain. Acte de la Réunion Phytosanitaire Coraf - Réseau Coton. 22-25 Février 2000 Lomé (Togo) : 209-218 pp.

7. Diabaté, D., Gnago, J.A., Koffi, K. \& Tano, Y. (2014). The effect of pesticides and aqueous extracts of Azadirachta indica (A.Juss.) and Jatropha carcus L. on Bemisia tabaci (Gennadius) (Homoptera: Aleyrididae) and Helicoverpa armigera (Hübner) (Lepidoptera: Noctuidae) found on tomato plants in Côte d'Ivoire. J. Appl. Biosci., 80, 7132- 7143.

8. Didi, G.J.R., Koné, P.W.E., Ochou, G.E.C., Dekoula, S.C., Kouakou, M., Bini, K.K.N.B., Yao, M.D.S., Mamadou, D. \& Ochou, O.G. (2018). Évolution spatio-temporelle des infestations de la mouche blanche Bemisia tabaci (Gennadius, 1889) associées à la culture cotonnière en Côte d'Ivoire. JABS. 121 : 12202-12210 URL: https://dx.doi.org/10.4314/jab.v121i1.10.

9. Doffou, N.M. (2012). Impact de la gestion de la résistance aux pyréthrinoïdes sur les populations de Cryptophlebia leucotreta Meyrick (Lepidoptera : tortricidae) et de Pectinophora gossypiella Saunders (lepidoptera : gelechiidae) en culture cotonnière en Côte d'Ivoire : de la sensibilité aux mécanismes. Thèse unique. Université Felix Houphouët-Boigny Cocody. Côte d'Ivoire. 178 p.

10. Doumbouya, M., Abo, K., Lepengue, A. N., Camara B., Kanko K., AIDARA D. \& Koné, D. (2012). Activités comparées in vitro de deux fongicides de synthèse et de deux huiles essentielles, sur des champignons telluriques des cultures maraîchères en Côte d'Ivoire. J. Appl. Biosci., 50: 3520 - 3532.

11. Fening, K.O., Adama, I. \& Tegbe, R.E. (2014). On-farm evaluation of homemade pepper extract in the management of pests of cabbage, Brassica oleraceae L., and french beans, Phaseolus vulgaris L., in two agro-ecological zones in Ghana. Afr. Entomol., 22(3), 552-560. 
12. Gonzalez, C. A., Reina, M., Diaz, C.E., Fraga, B.M. \& SantanaMeridas, O. (2013). Natural Product-Based Biopesticides for Insect Control. In: Reedijk, J., Ed. Elsevier Reference Module in Chemistry, Molecular Sciences and Chemical Engineering. Waltham, MA, Elsevier. 01-Nov-13. URL: http://dx.doi.org/10.1016/B978-0-12409547-2.02770-0.

13. Guèye, M.T., Seck, D., Wathelet, J.P. \& Lognay, G. (2011). Lutte contre les ravageurs des stocks de céréales et de légumineuses au Sénégal et en Afrique occidentale : synthèse bibliographique. Biotechnol. Agron. Soc. Environ., 15(1) : 183-194.

14. Hassane, S.O.S., Ghanmi, M., Satrani, B., Farah, A., Amarti, F., Achmet, S.M. \& Chaouch, A. (2011). Composition chimique et bioactivité des huiles essentielles de deux provenances de Ocimum canum S. de l'île de la Grande Comore. Phytothérapie, 9 : 18-24, DOI 10.1007/s10298-010-0602-5.

15. Johnson, F., Seri-Kouassi, B., Aboua, L. R. N. \& Foua-Bi, K. (2006). Utilisation de poudres et d'extraits totaux issus de plantes locales des genres ocimum sp. et mentha sp. comme biopesticides dans la lutte contre callosobruchus maculatus fab. Agronomie Africaine 18 (3) : 221-233.

16. Kaan, P., Ömer, C.K., Yasemin, Y., Salih, G., Betül, D., Fatih, D. \& Kemal, C.B. (2016). Insecticidal activity of Salvia veneris Hedge, essential oil against coleopteran stored product insects and Spodoptera exigua (Lepidoptera). Industrial Crops and Products, 97, 93-100. URL: https://doi.org/10.1016/j.indcrop.2016.12.012.

17. Kassi, M.F., Badou, J. O., Tonzibo, F. Z., Salah, Z., Bolou, A. B. B., Camara, B., Amari, E. G. D. N.-L. \& Koné, D. (2014). Potentiel antifongique de l'huile essentielle de Ocimum gratissimum dans la lutte biologique contre la maladie des raies noires du bananier causé par Mycosphaerella fijiensis morelet (mycosphaerellacea). Agronomie Africaine, 26 (2) : 1 - 11.

18. Koffi, A.M., Tonzibo, Z.F., Delort, L., Ruiz, N., Caldefie-Chézet, L. \& Chalchat, J.C. (2013). Corrélation entre la composition chimique et l'activité antifongique des huiles essentielles à prédominance thymol sur Candida albicans et Aspergillus fumigatus. Phytothérapie, 11 :134-139. DOI : 10.1007/s10298-013-0767-9.

19. Konan, N.O. \& Mergeai, G. (2007). Possibilités d'amélioration de la principale espèce cultivée de cotonnier (Gossypium hirsutum L.) pour la résistance au nématode réniforme (Rotylenchulus reniformis Linford et Oliveira). Biotechnol. Agron. Soc. Environ. 11 (2), 159171. 
20. Koné, P.W.E., Ochou, G.E.C., Didi, G.J.R., Dekoula, S.C., Kouakou, M., Bini, K.K.N.B., Mamadou, D. \& Ochou, O.G. (2017). Evolution spatiale et temporelle des dégâts du jasside Jacobiella facialis Jacobi, 1912 (Cicadellidae) en comparaison avec la distribution de la pluviométrie au cours des vingt dernières années dans les zones de culture cotonnière en Côte d'Ivoire. IJBCS. 11(3) : 1190-1201, URL : https://dx.doi.org/10.4314/ijbcs.v11i3.21.

21. Konstantopoulou, L., Vassilopoulou, L., Mauragani, T.P. \& Scouras, Z.G. (1992). Insecticidal effects of essential oils. A study of the effects of essential oils extracted from eleven Greek aromatic plants on $D$. auraria Experientia, 48 (6), 535-619. URL: https://doi.org/10.1007/BF01920251.

22. Koul, O., Rajwinder, S., Birpal, K. \& Dharamvir, K. (2013). Comparative study on the behavioral response and acute toxicity of some essential oil compounds and their binary mixtures to larvae of Helicoverpa armigera, Spodoptera litura and Chilo partellus. Insect Biopesticide Research Centre, 30 Parkash Nagar, Jalandhar 144003, Indiaa, Industrial Crops and Products, 49, 428-436. URL: https://doi.org/10.1016/j.indcrop.2013.05.032.

23. Martin, T., Ochou, O.G., Hala, N.F., Vassal, J.M. \& Vaissayre, M. (2000). Pyrethroid resistance in the cotton bollworm, Helicoverpa armigera (Hübner), in West Africa. Pest. Manag. Sci., 56 : 549-554. https://doi.org/10.1002/(SICI)1526-4998(200006)56:6<549::AIDPS160>3.0.CO;2-Y.

24. Ngamo, L.S., Ngassoum, M.B., Jirovetz, L., Ousman, A., Nukenine, C.E. \& Mukala, O.E. (2001). Protection du maïs stocké contre Sitophilus zeamais Motsch. Par l'utilisation d'huiles essentielles d'épices en provenance du Cameroun. Meded Rijks univ Gent Fak Landbouwkd Toegep Biol Wetensch., 66(2) : 473-478.

25. Nguemtchouin, M.G. (2012). Formulation d'insecticides en poudre par adsorption des huiles essentielles de Xylopia aethiopica et de Ocimum gratissimum sur des argiles camerounaises modifiées. Thèse de doctorat. ENSC de Montpellier, France, 293p.

26. Ouédraogo, I., Sawadogo, A., Nebie, R.CH. \& Dakouo, D. (2016). Evaluation de la toxicité des huiles essentielles de Cymbopogon nardus (L) et Ocimum gratissimum (L) contre Sitophilus zeamais Motsch et Rhyzopertha dominica F, les principaux insectes nuisibles au maïs en stockage au Burkina Faso. Int. J. Biol. Chem. Sci. 10(2): 695-705, 2016. DOI: http://dx.doi.org/10.4314/ijbcs.v10i2.20

27. Patana, R. (1977). Layered Diet for Pink Bollworm Rearing, U.S. Departement of Agriculture, Agricultural Research Service, ARS W47, $10 \mathrm{p}$. 
28. Priestley, C.M., Williamson, E.M., Wafford, K.A. \& Sattelle, D.B. (2003). Thymol, a constituent of thyme essential oil, is a positive allosteric modulator of human GABA receptors and a homooligomeric GABA receptor from Drosophila melanogaster. Br. J. Pharmacol., 140, 1363-1372. URL : https://doi.org/10.1038/sj.bjp.0705542.

29. Saunders, W. W. (1843). Description of a species of moth destructive to the cotton crops in India. Transactions of the entomological Society of London 1844, Catalogue of Life.

30. Soro, S., Abo, K., Koné, D., Coffi, K., Kouadio, J. Y. \& Ake, S. (2011). Comparaison de l'efficacité antifongique de l'huile essentielle d'ocimum gratissimum L. et du fongicide de synthèse mancozèbe contre le mycopathogène tellurique, fusarium oxysporum f. sp. Radicis-lycopersici en cultures de tomate (lycopersicon esculentum mill.) sous abri en côte d'Ivoire. Agronomie Africaine 23 (1) : 43 - 52 URL: https://www.researchgate.net/publication/230735049.

31. Tchoumbougnang, F., Dongmo, J. P. M., Sameza, M.L., Mbanjo, N. E.G., Fotso, G.B.T., Amvam, Z. P. H. \& Menut, C. (2009). Activité larvicide sur Anopheles gambiae Giles et composition chimique des huiles essentielles extraites de quatre plantes cultivées au Cameroun, BASE [En ligne], 1 (13),77-84. URL : http://popups.ulg.ac.be/17804507/index.php?id=3547.

32. Tia, E.V., Lozano, P., Lozano, Y.F., Martin, T., Niamké, S. \& Adima, A.A. (2013). Potentialité des huiles essentielles dans la lutte biologique contre la mouche blanche Bemisia tabaci Genn. Phytothérapie, 11 (1), 31-38. URL : https://doi.org/10.1007/s10298012-0736-8.

33. Traore, S. K., Dembele, A., Kone, M., Mambo, V., Lafrance, P., Bekro, Y-A. \& Houenou, P. (2008). Contrôle des pesticides organochlorés dans le lait et produits laitiers : Bioaccumulation et Risques d'exposition. Afrique Sciences, 04(1), 87-98. URL : http://dx.doi.org/10.4314/afsci.v4i1.61660.

34. Traore, O., Sereme, A., Dabire, C. M., Some, K. \& Nebie, R. H. C. (2015). Effet des extraits du thé de Gambie (Lippia multiflora Moldenk) et du neem (Azadirachta indica A. Juss.) sur Helicoverpa armigera et les thrips de la tomate (Lycopersicon esculentum Mill.). J. Appl. Biosci., 95, 8930-8936.

35. Wu, H. H., Wu, K. M., Wang, D. Y. \& Guo, Y. Y. (2006). Flight potential of pink bollworm, Pectinophora gossypiella (Lepidoptera: Gelechiidae). Environmental Entomology, 35: 887-893. URL: https://doi.org/10.1603/0046-225X-35.4.887. 
36. Wu, H.H., Huang, M.S., Wan, P., Kris, A G.W. \& Wu, K.M. (2013). Emergence, Mating and Oviposition Behavior of the Chinese Population in Pink Bollworm, Pectinophora gossypiella (Lepidoptera: Gelechiidae). Journal of Integrative Agriculture, 12(4), 653-662. URL: https://doi.org/10.1016/S2095-3119(13)60283-X

37. Yarou, B. B., Silvie, P., Komlan, F. A., Mensah, A., Alabi, T., Verheggen, F., Francis F. (2017). Plantes pesticides et protection des cultures maraîchères en Afrique de l'Ouest (synthèse bibliographique). Biotechnol. Agron. Soc. Environ. 2017 21(4), 288304. 